Pacific Journal of Mathematic 


\title{
ON THE STRUCTURE OF ALGEBRAIC ALGEBRAS
}

\author{
ThOMAS J. LAFFEY
}

Let $A$ be an infinite dimensional (associative) algebra over a field $F$. It is shown that $A$ has an infinite dimensional commutative subalgebra $C$ of one of the following types:

(a) $C$ is generated by one element

(b) $C^{2}=\{0\}$

(c) $C^{3}=\{0\}$ and $C$ is an ideal of an ideal of $A$

(d) $C$ is generated by mutually orthogonal idempotents

(e) $C$ is a field.

A necessary and sufficient condition (in terms of the quadratic forms over $F$ ) is obtained for the validity of the statement: Every infinite dimensional nil algebra over $F$ has an infinite dimensional subalgebra $B$ with $B^{2}=\{0\}$. An ideal $y(A)$ of an algebra $A$ (analogous to the F.C. subgroup in group theory) is defined and several properties of it are obtained.

In $\S 1$ of this paper we give a list of notation and definitions. In $\$ 2$ we prove some preliminary results on rings and algebras. In $\$ 3$ we obtain several properties of an ideal $y(A)$ of an algebra $A$ (defined in $\$ 1$ ). The ideal $y(A)$ is an algebra-theoretic analogue of the F.C. (finite conjugate) subgroup in group theory. The main result of this paper is the Main Theorem, proved in $\$ 4$, which states:

MAIN Theorem (4.1). Let $A$ be an infinite dimensional (associative) algebraic algebra over a field $F$ and assume

(a) A contains no infinite set of mutually orthogonal idempotents.

(b) A contains no infinite dimensional subalgebra $B$ with $B^{2}=0$

(c) $y(A)$ is finite dimensional.

Then $A=M \oplus N$ where $M$ is finite dimensional and $N$ is the direct sum of finitely many algebraic division algebras.

An immediate consequence of the Main Theorem is the fact, proved in [5], that every infinite dimensional associative algebra over a field has an infinite dimensional commutative subalgebra.

Several results in this paper deal with algebraic algebras which have no infinite dimensional subalgebras with square zero. This condition restricts the structure of an algebraic algebra considerably. For example, if $A$ is a nil algebra over a field and $A$ has no infinite dimensional subalgebra $C$ with $C^{2}=0$, then $A$ is locally finite and $A$ is even necessarily finite dimensional for a large class of fields. 
We wish to thank the referee of an earlier draft of this paper for several useful comments which have helped to simplify the proofs and improve the presentation of the paper considerably.

We also wish to thank the Mathematical Sciences Division of Northern Illinois University for their hospitality during the session 1972-73 when this work was carried out.

1. Notation and definitions. Throughout the remainder of the paper the words ring and algebra will mean associative ring and associative algebra, respectively. If $A$ is an algebra over a field and $B$ is a subspace (or subalgebra) of $A, A / B$ denotes the factor space of $A$ by $B$ and, if $B$ is an ideal of $A, A / B$ denotes the corresponding factor algebra.

If $X, Y$ are subspaces or subalgebras of $A, X+Y$ denotes the vector space sum of $X, Y$. If $X$ or $Y$ is an ideal of $A$, then $X+Y$ coincides with the algebra sum of $X, Y$. Also $\operatorname{dim}(X)$ denotes the dimension of $X$.

$\oplus$ is used to denote the direct sum of algebras.

If $S$ is a nonempty subset of ring $R$, then ring $(S)$ denotes the subring generated by $S$. If $B$ is a nonempty subset of an algebra $A$, alg $(B)$ denotes the subalgebra generated by $A$.

If $S$ is a nonempty subset of ring $R$, then

$$
\operatorname{Ann}(S)=\{r \in R \mid r s=s r=0 \text { for all } s \in S\} \text {. }
$$

If $S=\{x\}$, write Ann $(\{x\})=\operatorname{Ann}(x)$.

If $A$ is an algebra, we write

$$
y(A)=\{a \in A / \operatorname{dim}(A / \operatorname{Ann}(a)) \text { is finite }\}
$$

An algebra $A$ is called algebraic over a field $F$ if each element of $A$ generates a finite dimensional subalgebra $A . \quad A$ is called locally finite if each finite nonempty subset of $A$ generates a finite dimensional subalgebra of $A$.

We recall that by a result of Golod-Shafarevich ([3], Theorem (8.1.3)) there exist algebras which are algebraic but not locally finite.

We say that a field $F$ is square-dependent if given any sequence $\left\{a_{n}\right\}$ of elements of $F$, there exists a positive integer $N=N\left(\left\{a_{n}\right\}\right)$ and elements $\lambda_{1}, \cdots, \lambda_{N} \in F$, not all zero, such that

$$
a_{1} \lambda_{1}^{2}+\cdots+a_{N} \lambda_{N}^{2}=0 .
$$

We note that a formally real field is not square-dependent.

Several results on and examples of square-dependent fields can be found in [2].

0 is used to denote both the zero element and the zero algebra. 
2. Some general results on algebras. Our first result deals with subalgebras of finite codimension. The group-theoretic analogue of this result is well-known and the ring-theoretic analogue has been obtained by J. Lewin ([6]).

Lemma (2.1). Let $A$ be an algebra over a field $F$ and let $B$ be a subalgebra of $A$ with $\operatorname{dim}(A / B)=n<\infty$. Then $B$ contains an ideal I of $A$ with $\operatorname{dim}(A / I) \leqq n(n+1)^{2}$.

Proof. Let $x_{1}+B, \cdots, x_{n}+B$ be a basis for $A / B$. Define subspaces $B_{i j}$ of $B$ as follows:

Let

$$
\begin{aligned}
B_{\imath 0} & =\left\{b \in B \mid x_{\imath} b \in B\right\} & & (i \geqq 1) \\
B_{0 \jmath} & =\left\{b \in B \mid b x_{\jmath} \in B\right\} & & (j \geqq 1) \\
B_{i j} & =\left\{b \in B \mid x_{\imath} b x_{\jmath} \in B\right\} & & (i \geqq 1, j \geqq 1) .
\end{aligned}
$$

Fix $(i, j) \neq(0,0)$. The map $b \rightarrow x_{i} b x_{\jmath}+B(b \in B)$ is an $F$-space homomorphism of $B$ into $A / B$ with kernel $B_{i j}$. So $\operatorname{dim}\left(B / B_{i j}\right) \leqq n$. Let $I=\bigcap_{(i, j) \neq(0,0)} B_{i j \cdot}$. Then

$$
\operatorname{dim}(A / I)=\operatorname{dim}(A / B)+\operatorname{dim}(B / I) \leqq n+n\left((n+1)^{2}-1\right)=n(n+1)^{2} .
$$

It is straightforward to check that $I$ is an ideal of $A$.

We are grateful to the referee and to H. E. Bell for shortening our original proof of the next result. The proof here is due to H. E. Bell.

Lemma (2.2). Let $R$ be a ring. The following statements are equivalent :

(1) $R$ has an infinite set of commuting idempotents.

(2) $R$ has an infinite set of mutually orthogonal idempotents.

Proof. Clearly (2) implies (1). Suppose then that (1) holds. Let $X$ be an infinite set of nonzero commuting idempotents of $R$. We may assume $R$ is generated by $X$. For each subring $S$ of $R$, let $E(S)$ be the set of nonzero idempotents in $S$. Note that if $e \in E(S)$, then $S=$ $e S+\operatorname{Ann}(e)$ so each $f \in E(S)$ splits as a sum $f_{1}+f_{2}$ of idempotents $f_{1}$, $f_{2}$. Hence if $E(S)$ is infinite so is at least one of $E(e S), E(\operatorname{Ann}(e))$. Thus starting with $E(R)$ we may construct a sequence $e_{1}, e_{2}, \cdots, e_{n}, \cdots$ of distinct nonzero idempotents such that for each $i$ either (a) $e_{i} e_{1}=0$ for all $j>i$ or (b) $e_{1} e_{1}=e_{1}$ for all $j>i$. If (a) holds for infinitely many $i$, then (2) follows. We may thus assume that (b) holds for all $i$. Then $\left\{e_{1}-e_{2}, e_{2}-\right.$ $\left.e_{3}, e_{3}-e_{4}, \cdots\right\}$ is an infinite set of mutually orthogonal idempotents. 
COROllaRY (2.3). Let $R$ be a ring with no nonzero nilpotent elements. The following are equivalent:

(i) $R$ has an infinite set of mutually orthogonal idempotents.

(ii) $R$ has infinitely many idempotents.

Our next result gives some information on the structure of algebraic algebras.

Lemma (2.4). Let $A$ be an algebraic algebra over a field $F$ and assume that $A$ has no nonzero nilpotent elements. Then one of the following statements holds:

(i) A has an infinite set of mutually orthogonal idempotents.

(ii) $A$ is a finite direct sum of algebraic division algebras.

Proof. Assume that (i) does not hold. By Corollary (2.3), $A$ has only finitely many idempotents. Let $X$ be the subalgebra generated by the idempotents and let $e_{1}, e_{2}, \cdots, e_{n}$ be the primitive idempotents of $X$. Then every idempotent in $A$ is a sum of some of the $e_{i}$. If $a \in A$, the algebra generated by $a$ is finite dimensional and thus it is a direct sum of finitely many fields. Thus $a=e a$ for some idempotent $e$. Thus $A=e_{1} A+\cdots+e_{n} A$ and, since each $e_{i}$ is central and $e_{\imath} e_{j}=0(i \neq j)$, this sum is direct. Since $e_{i}$ is the only nonzero idempotent in $e_{t} A, e_{i} A$ is a division algebra. This completes the proof.

3. Properties of $y(A)$. In this section we obtain several properties of $y(A)$ for $A$ an algebra over a field $F$.

We begin with some elementary facts.

Lemma (3.1). Let $A$ be an algebra over a field $F$. Then

(i) $y(A)=A$ if $A$ in finite dimensional.

(ii) $y(y(A))=y(A)$.

(iii) if $a \in A$, then $a \in y(A)$ if and only if $A a+a A$ is a finite dimensional subspace of $A$.

(iv) if $B$ is a subalgebra of $A$ with $A / B$ finite dimensional, then $y(B)=B \cap y(A)$.

(v) if $y(A)$ is finite dimensional, then $y(A / y(A))=0$.

An easy consequence of Lemma (3.1)(iii) is.

Lemma (3.2). Let $A$ be an algebra over a field $F$. Then $y(A)$ is an ideal of $A$.

We now prove

Lemma (3.3). Let $A$ be an algebra over a field $F$. The following are equivalent. 
(a) $\quad a \in y(A)$

(b) $a$ is contained in a finite dimensional ideal of $A$.

Let $a \in y(A)$. Now $a A, A a$ are finite dimensional and also, since $a A \subseteq y(A), A a \subseteq y(A), A a A$ is finite dimensional. It follows easily that the smallest ideal containing $a$ is finite dimensional. The converse is trivial.

Corollary (3.4). Let $A$ be an algebra over a field $F$. Then $y(A)$ is a locally finite ideal of $A$.

Our next result gives some information on the size of $y(A)$.

LEMMA (3.5). Let $A$ be an algebra over a field $F$ and assume that $A$ has no infinite dimensional subalgebra $C$ with $C^{2}=0$. Then $a \in y(A)$ if and only if $a^{2} \in y(A)$.

Proof. Let $a \in A$ be such that $a^{2} \in y(A)$. The map $x \rightarrow$ axa $\left(x \in \operatorname{Ann}\left(a^{2}\right)\right)$ is a linear transformation of $\operatorname{Ann}\left(a^{2}\right)$ whose image is a subalgebra of $A$ whose square is zero. Thus

$$
U=\left\{x \in \operatorname{Ann}\left(a^{2}\right) \mid a x a=0\right\}
$$

has finite codimension in $\operatorname{Ann}\left(a^{2}\right)$ and therefore in $A$. Let $W=$ $\{a x \mid x \in U\}$. Then $W$ is a subalgebra of $A$ whose square is zero. So $W$ is finite dimensional. Thus

$$
V=\{x \in U \mid a x=0\}
$$

has finite codimension in $U$. Using symmetry, we find that $a \in y(A)$.

The converse is trivial.

Corollary (3.6). Let $A$ be an algebra over a field $F$ and assume that $A$ has no infinite dimensional subalgebra whose square is zero. Then $A / y(A)$ has no nonzero nilpotent elements.

COROllary (3.7). Let $A$ be a nil algebra over a field $F$ and assume that $A$ has no nonzero finite dimensional ideals. Then $A$ has an infinite dimensional subalgebra whose square is zero (unless $A=0$ ).

Lemma (3.8). Let $A$ be an algebra over a field $F$ and assume that $y(A)$ is infinite dimensional. Then there exists a sequence $\left\{X_{n} \mid n=\right.$ $1,2,3, \cdots\}$ of ideals of $A$ such that 
and

(a) $X_{n}$ is finite dimensional.

(b) $X_{n} \subseteq y(A)$

(c) $X_{n} X_{m}=0(m \neq n)$

(d) $X_{n+1} \not \subset X_{1}+\cdots+X_{n}(n \geqq 1)$.

Proof. Note if $x \in y(A)$ then the smallest ideal of $A$ containing $x$ is finite dimensional. Having chosen elements $x_{1}, \cdots, x_{n} \in y(A)$ such that if $X_{i}$ is the smallest ideal of $A$ containing $x_{i}$, then

$$
\begin{aligned}
& \text { ( } \alpha) X_{t+1} \not \subset X_{1}+\cdots+X_{\iota} \quad(i \geqq 1) \\
& \text { ( } \beta) X_{\imath} X_{\jmath}=0 \quad(i \neq j)
\end{aligned}
$$

let

$$
W_{n}=y(A) \cap \operatorname{Ann}\left(X_{1}+\cdots+X_{n}\right) .
$$

Note that $W_{n}$ is infinite dimensional and that $X_{1}+\cdots+X_{n}$ is finite dimensional. Let

$$
x_{n+1} \in W_{n}-\left(X_{1}+\cdots+X_{n}\right)
$$

and let $X_{n+1}$ be the smallest ideal of $A$ containing $x_{n+1}$. The result follows by induction.

Lemma (3.9). Let $F$ be a square dependent field, $V$ a finite dimensional $F$-space and $v_{0}, v_{1}, v_{2}, \cdots$ an infinite sequence of elements of $V$. Then there exist sequences $\lambda_{0}, \lambda_{1}, \lambda_{2}, \cdots$ of elements of $F$ and $0=n(0)<$ $n(1)<n(2)<\cdots$ natural numbers, such that and

(a) for all $j$, there exists $i$ with $n(j) \leqq i<n(j+1)$ such that $\lambda_{i} \neq 0$,

(b) $\sum_{n(j) \leqq i<n(j+1)} \lambda_{\imath}^{2} v_{\imath}=0$.

Proof. If $\operatorname{dim} V=1$, the result is immediate from the definition of a square-dependent field. Suppose $\operatorname{dim} V=n>1$ and let $W$, spanned by $w$, be a one-dimensional subspace. Using induction we may assume that there exist sequences $\lambda_{0}, \lambda_{1}, \lambda_{2}, \cdots$ of elements of $F$ and $0=n(0)<$ $n(1)<n(2)<\cdots$ of natural numbers such that

(a) for all $j$, there exists $i$ with $n(j) \leqq i<n(j+1)$ such that $\lambda_{i} \neq 0$ and

(b) $\sum_{n(j) \leqq i<n(j+1)} \lambda_{i}^{2} v_{i}=\alpha_{j} w$ for some $\alpha_{j} \in F$.

Again, by the definition of a square-dependent field, there exist sequences $\mu_{0}, \mu_{1}, \mu_{2}, \cdots$ of elements of $F$ and $0=m(0)<m(1)<m(2)$ $<\cdots$ of natural numbers such that 

and

(c) for all $j$, there exists $i$ with $m(j) \leqq i<m(j+1)$ such that $\mu_{i} \neq 0$

(d) $\quad \sum_{m(j) \leqq i<n(j+1)} \mu_{i}^{2} \alpha_{i}=0$.

Hence

$$
\sum_{m(k) \leqq j<m(k+1)} \sum_{n(j) \leqq i<n(j+1)} \lambda_{\imath}^{2} \mu_{j}^{2} v_{\imath}=0
$$

and the result follows.

THEOREM (3.10). Let $A$ be an algebra over a field $F$ and assume that $y(A)$ is infinite dimensional. Then one of the following statements holds:

(a) $y(A)$ contains an infinite set of mutually orthogonal idempotents.

(b) A has an ideal I such that

(i) $I^{3}=\{0\}$

(ii) I has an infinite dimensional commutative ideal $C$. If, in addition, $F$ is square-dependent, statement

(b)(ii) can be improved to

(b)(ii)' $y(A)$ contains an ideal I of $A$ such that I has an infinite dimensional ideal $C$ with $C^{2}=0$.

Proof. Let $\left\{X_{n}\right\}_{1}^{\infty}$ be an infinite sequence of ideals of $A$ satisfying the conclusion of Lemma (3.8). If infinitely many of the $X_{i}$ contain nonzero idempotents, then conclusion (a) of the theorem holds. Assume then that only finitely many of the $X_{i}$ contain nonzero idempotents. By omitting these and relabelling, if necessary, we may assume that no $X_{\imath}$ contains a nonzero idempotent and further that $X_{i}$ is nilpotent for all $i$.

Let $k_{l}$ be the least positive integer for which $X_{i}^{k_{i}}=0$ and let $h_{i}=k_{i} / 2$ (if $k_{i}$ is even), $h_{i}=\left(k_{i}+1\right) / 2$ (if $k_{i}$ is odd). Let $H_{i}=X_{i}^{h_{i}}$. Now $H_{i}$ is an ideal of $A$ and $H_{t}^{2}=0$. Also $H_{t} H_{l}=0$. Let $H$ be the algebra generated by $\left\{H_{1}\right\}$. Then $H^{2}=0, H$ is an ideal of $A$ and, if $H$ is infinite dimensional, conclusion (b) of the theorem holds with $I=C=H$.

Assume from now on that $H$ is finite dimensional. There exists a positive integer $m$ such that for all $n>m$,

$$
H_{n} \subseteq H_{1}+\cdots+H_{m}
$$

In particular $X_{n} H_{n}=H_{n} X_{n}=0$. Thus $X_{n}^{3}=0$ for all $n>m$. Let $I$ be the ideal generated by $\left\{X_{n}+H \mid n>m\right\}$. Then $I^{3}=0$ and conclusion (b) (i) of the theorem holds.

Let $v_{n} \in X_{n}-\left(X_{m+1}+\cdots+X_{n-1}+H\right)(n>m)$ and let $C_{n}$ be the algebra generated by $\left\{v_{n}, H_{n}\right\}$. (We note that $v_{n}$ exists because of our choice of $m$ ). Since $X_{n} H=H X_{n}=0, C_{n}$ is commutative. Also $C_{n}$ is an 
ideal of $I$. Thus conclusion (b) (ii) of the theorem holds with $C=$ $\operatorname{alg}\left(\left\{C_{n}\right\}\right)$.

Suppose now that $F$ is a square-dependent field. Let $v_{n}^{2}=$ $h_{n} \in H$. Since $H$ is finite dimensional, Lemma (3.9) provides us with a sequence $\lambda_{0}, \lambda_{1}, \cdots$ of elements of $F$ and $0=n(0)<n(1)<n(2)<\cdots$ of natural numbers such that and

(1) for all $j$, there exists $i$ with $n(j) \leqq i<n(j+1)$ such that $\lambda_{l} \neq 0$

(2) $\sum_{n(J) \leqq i<n(J+1)} \lambda_{t}^{2} h_{t}=0$.

Let $w_{j}=\sum_{n()) \leqq i<n(j+1)} \lambda_{i} v_{i}$.

Then $w_{1} w_{k}=0$ for all $j, k$ and $\left\{w_{j}\right\}$ is linearly independent. Also the algebra generated by $H \cup\left\{w_{j}\right\}$ is an ideal of $I$. Thus conclusion (b) (ii)' of the theorem holds. The proof is now complete.

EXAMPLE (3.11). Let $F$ be a field which is not square-dependent (i.e., there exists an infinite sequence $\left\{a_{n}\right\}_{1}^{\infty}$ of elements of $F$ such that

$$
a_{1} \lambda_{1}^{2}+\cdots+a_{m} \lambda_{m}^{2}=0
$$

for $\lambda_{i} \in F$ and $m \geqq 1$ implies $\lambda_{1}=\cdots=\lambda_{m}=0$ ). Let $A$ be the algebra generated over $F$ by

$$
u_{0}, u_{1}, \cdots, u_{n}, \cdots
$$

subject to the relations

$$
\begin{aligned}
& u_{0}^{2}=0, \quad u_{\imath}^{2}=a_{i} u_{0} \quad(i \geqq 1) \\
& u_{i} u_{j}=0 \quad(i \neq j) .
\end{aligned}
$$

Then $y(A)=A$ and $A u_{0}$ is the only subalgebra of $A$ whose square is zero. Thus the conclusion (b)(ii) cannot in general be modified to $C^{2}=0$ for fields which are not square-dependent.

Theorem (3.10) and Example (3.11) yield the following characterization of square-dependent fields.

THEOREM (3.12). Let $F$ be a field. The following are equivalent

(i) $F$ is square dependent

(ii) every infinite dimensional nil algebra over $F$ has an infinite dimensional subalgebra $C$ with $C^{2}=0$.

4. The main results. We first prove the Main Theorem.

Proof. Assume that $A$ satisfies (a), (b) and (c). By Lemma (3.1) (v), $y(A / y(A))=0$. By Corollary (3.6) and the fact that (b) holds, 
$A / y(A)$ has no nonzero nilpotent elements. Let $H=\operatorname{Ann}(y(A))$ and $L=y(A) \cap H$. Then $H / L$ is isomorphic to a subalgebra of $A / y(A)$, so $H / L$ has no nonzero nilpotent elements. Next we show that $H / L$ does not have an infinite set of mutually orthogonal idempotents. For suppose that $\left\{f_{n}+L\right\}$ is such a set. Then, since $H L=L H=0,\left\{f_{n}^{2}\right\}$ is an infinite set of mutually orthogonal idempotents of $A$, against our assumptions.

Now Lemma (2.4) implies that $H / L$ is a finite direct sum of division algebras. In particular $H / L$ has an identity $e+L$. Let

$$
K=\left\{w \in H \mid w e^{2}=e^{2} w=w\right\}
$$

Since $w e^{2}-w$ and $e^{2} w-w$ are elements of $L$ for all $w \in H$, we see that $K$ has finite codimension in $H$. Also $f=e^{2}$ is the identity of $K$. Let

$$
N_{0}=\{a \in A \mid a f=f a=a\} .
$$

We note that $A / N_{0}$ is finite dimensional. Let $a \in A$ and consider $a f-a$. Then $(a f-a) K_{1}=K_{1}(a f-a)=0$ where $K_{1}$ is the maximal ideal of $A$ contained in $K$. Thus $a f-a \in y(A)$. Similarly $f a-a \in$ $y(A)$. Now

$$
a=f a f+(a f-f a f)+(a-a f) \in N_{0}+y(A) .
$$

Let $N$ be the maximal ideal of $A$ contained in $N_{0}$. Again, by Lemma (2.1), $A / N$ is finite dimensional. Since $f \neq 0$ and $f y(A)=0$, $N_{0} \cap y(A)=0$. So $N$ is isomorphic to a subalgebra of $H / L$. In particular $N$ is a finite direct sum of division algebras. So $N$ has an identity, $g$ say. Now if $u \in A, u g-u$ is annihilated by $N$. Thus $u g-u \in y(A)$. Thus $u \in y(A)+N=y(A) \oplus N$.

This completes the proof for general $F$.

Note that if $F$ is square-dependent, (c) is a consequence of (a), (b), by Theorem (3.12).

Since a maximal subfield of an infinite dimensional division algebra is infinite dimensional ([4], p. 165), Theorem (4.1) implies

COROLlaRy (4.2). Every infinite dimensional algebra over a field $F$ has an infinite dimensional commutative subalgebra.

This is the main result of [5]. We note that Theorem (4.1) shows that such a commutative subalgebra $C$ can be found of one of the following types:

(a) $C$ is generated by one element. 
(b) $C^{2}=0$.

(c) $C$ is generated by mutually orthogonal idempotents.

(d) $C^{3}=0$ and $C$ is an ideal of an ideal of $A$.

(e) $C$ is a field.

We now prove

THEOREM (4.3). Let $A$ be an algebraic algebra over a field $F$ with $F$ finite or algebraically closed. If $A$ is not locally finite, then $A$ has an infinite dimensional subalgebra $C$ with $C^{2}=0$.

Proof. Suppose that the theorem is false and let $A$ be a counterexample. By Corollary (3.6), $A / y(A)$ has no nonzero nilpotent elements. Suppose first that $F$ is finite. Let $x \in A / y(A)$. The ring generated by $x$ is finite (since $A$ is algebraic) and has no nonzero nilpotent elements, so $x^{p^{k}}=x$ for some $k \geqq 1$ where $p$ is the characteristic of $F$. Then $A / y(A)$ is commutative by Jacobson's theorem ([3], Theorem (3.1.2)) and thus it is locally finite. Hence $A$ is locally finite, since $y(A)$ is locally finite.

Suppose then that $F$ is algebraically closed. Each element $x \in$ $A / y(A)$ is then an $F$-linear combination of idempotents all of which are central, since $A / y(A)$ has no nonzero nilpotent elements. So $A / y(A)$ is commutative and the result follows.

Our next result is a consequence of the proof of Theroem (3.10), and the Main Theorem.

TheOrem (4.4). Let $A$ be an algebraic algebra over a field $F$ and assume that $A$ has no infinite dimensional subalgebra $C$ with $C^{2}=0$. Then $A$ is Artinian if and only if $A$ is Noetherian.

Proof. If $A$ is Noetherian, then the proof of Theorem (4.1) shows that conclusion (iv) of Theorem (4.1) must occur. Thus $A$ is Artinian.

Suppose now that $A$ is Artinian and that $A$ is not Noetherian. Clearly conclusion (i) or (iv) of Theorem (4.1) cannot occur, so conclusion (iii) must occur. Thus $y(A)$ is infinite dimensional. Choose the sequence $\left\{X_{n}\right\}$ as in Lemma (3.8). Then the sequence

$$
\operatorname{Ann}\left(X_{1}\right), \quad \operatorname{Ann}\left(X_{1}+X_{2}\right), \cdots, \operatorname{Ann}\left(X_{1}+\cdots+X_{n}\right), \cdots
$$

does not terminate.

We give two final results on how the assumption that an algebra $A$ has no infinite dimensional subalgebra whose square is zero restricts the structure of $A$. These results are elementary. 
THEOREM (4.5). Let $A$ be an algebra over a field $F$ and assume that $A$ has no infinite dimensional subalgebra $B$ with $B^{2}=0$. Then

(a) every left invertible element of $A$ is right invertible,

(b) if $A$ has an element which is a left zero-divisor but is not a right zero-divisor, then $A$ has a finite dimensional nonzero ideal I with $I^{2}=0$.

Proof. (a) Suppose $x, y \in A$ with $y x=1, x y \neq 1$. Suppose that $\left\{x^{k}(x y-1) \mid k \geqq 1\right\}$ is linearly dependent. Let $\lambda_{0}, \cdots, \lambda_{k} \in F, \lambda_{k} \neq 0$, be such that

$$
\lambda_{0}(x y-1)+\lambda_{1} x(x y-1)+\cdots+\lambda_{k} x^{k}(x y-1)=0 .
$$

Multiplying on the left by $y^{k}$, we get $x y-1=0$, which is impossible.

(b) Let $z \in A$ be such that $z x=0$ for some $0 \neq x \in A$ and that $u z=0$ for $u \in A$ implies $u=0$. The subalgebra $C$ generated by the elements $x z^{k}(k=1,2, \cdots)$ satisfies $C^{2}=0$, so it is finite dimensional. Then since $z$ is not a right zero-divisor it follows that $\left\{x z^{k} \mid k=\right.$ $0,1,2, \cdots\}$ is linearly dependent. Hence there exist $a_{0}, a_{1}, \cdots, a_{m} \in F$, not all zero, such that

$$
a_{0} x+a_{1} x z+\cdots+a_{m} x z^{m}=0 .
$$

We may assume that $m$ is the least such integer. Then $a_{0} \neq 0$. Hence $x^{2}=0$. By Corollary (3.6), $x \in y(A)$. The same argument shows that $w^{2}=0$ for all $w$ in the smallest right ideal of $A$ containing $x$. Thus, for $c \in A$,

$$
0=(x+x c)^{2}=x^{2}+x^{2} c+x c x+(x c)^{2}=x c x .
$$

Hence $(A x)^{2}=(x A)^{2}=0$. The result follows.

\section{REFERENCES}

1. Z. I. Borevich, I. R. Shafarevich, Number Theory, Academic Press, New York, 1966.

2. M. Greenberg, Lectures on forms in many variables; Benjamin, New York.

3. I. N. Herstein, Noncommutative rings, Carus Math. Monograph, No. 15, Math. Assod. of Amer., 1968.

4. N. Jacobson, Structure of rings, Amer. Math. Soc. Colloquium Publ. XXXVII, (1964).

5. T. J. Laffey, Commutative subalgebras of infinite dimensional algebras, Bull. London Math. Soc., 5 (1973), 312-314.

6. J. Lewin, Subrings of finite index in finitely generated rings, J. Algebra, 5 (1967), 84-88.

Received March 28, 1973 and in revised form January 13, 1976. 



\section{PACIFIC JOURNAL OF MATHEMATICS}

\section{EDITORS}

RICHARD ARENS (Managing Editor)

University of California

Los Angeles, California 90024

\section{J. Dugundu}

Department of Mathematics University of Southern California Los Angeles, California 90007

D. Gilbarg and J. Milgram Stanford University Stanford, California 94305

\section{ASSOCIATE EDITORS}
E. F. BECKENBACH
B. H. NeumanN
F. WOLF
K. YoSHIDA

\section{SUPPORTING INSTITUTIONS}

UNIVERSITY OF BRITISH COLUMBIA CALIFORNIA INSTITUTE OF TECHNOLOGY

UNIVERSITY OF CALIFORNIA

MONTANA STATE UNIVERSITY

UNIVERSITY OF NEVADA

NEW MEXICO STATE UNIVERSITY

OREGON STATE UNIVERSITY

UNIVERSITY OF OREGON

OSAKA UNIVERSITY

\author{
UNIVERSITY OF SOUTHERN CALIFORNIA \\ STANFORD UNIVERSITY \\ UNIVERSITY OF HAWAII \\ UNIVERSITY OF TOKYO \\ UNIVERSITY OF UTAH \\ WASHINGTON STATE UNIVERSITY \\ UNIVERSITY OF WASHINGTON \\ AMERICAN MATHEMATICAL SOCIETY
}

The Supporting Institutions listed above contribute to the cost of publication of this Journal, but they are not owners or publishers and have no responsibility for its contents or policies.

Mathematical papers intended for publication in the Pacific Journal of Mathematics should be in typed form or offset-reproduced (not dittoed), double spaced with large margins. Underline Greek letters in red, German in green, and script in blue. The first paragraph or two must be capable of being used separately as a synopsis of the entire paper. Items of the bibliography should not be cited there unless absolutely necessary, in which case they must be identified by author and Journal, rather than by item number. Manuscripts, in duplicate, may be sent to any one of the four editors. Please classify according to the scheme of Math. Reviews, Index to Vol. 39. All other communications should be addressed to the managing editor, or Elaine Barth, University of California, Los Angeles, California, 90024.

100 reprints are provided free for each article, only if page charges have been substantially paid. Additional copies may be obtained at cost in multiples of 50 .

The Pacific Journal of Mathematics is issued monthly as of January 1966. Regular subscription rate: $\$ 72.00$ a year (6 Vols., 12 issues). Special rate: $\$ 36.00$ a year to individual members of supporting institutions.

Subscriptions, orders for back numbers, and changes of address should be sent to Pacific Journal of Mathematics, 103 Highland Boulevard, Berkeley, California, 94708.

PUBLISHED BY PACIFIC JOURNAL OF MATHEMATICS, A NON-PROFIT CORPORATION

Printed at Jerusalem Academic Press, POB 2390, Jerusalem, Israel.

$$
\begin{gathered}
\text { Copyright } 1976 \text { Pacific Journal of Mathematics } \\
\text { All Rights Reserved }
\end{gathered}
$$




\section{Pacific Journal of Mathematics}

Vol. 62, No. 2

February, 1976

Allan Russell Adler and Catarina Isabel Kiefe, Pseudofinite fields, procyclic

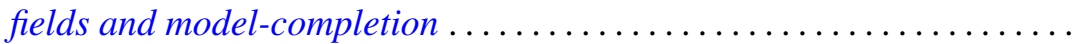

Christopher Allday, The stratification of compact connected Lie group

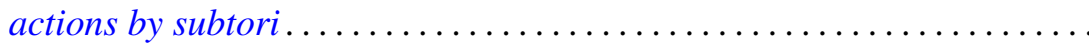

Martin Bartelt, Commutants of multipliers and translation operators .......

Herbert Stanley Bear, Jr., Ordered Gleason parts ..................

James Robert Boone, On irreducible spaces. II .....................

James Robert Boone, On the cardinality relationships between discrete

collections and open covers ............................

L. S. Dube, On finite Hankel transformation of generalized functions .......

Michael Freedman, Uniqueness theorems for taut submanifolds . . . . . . . . .

Shmuel Friedland and Raphael Loewy, Subspaces of symmetric matrices

containing matrices with a multiple first eigenvalue .............

Theodore William Gamelin, Uniform algebras spanned by Hartogs

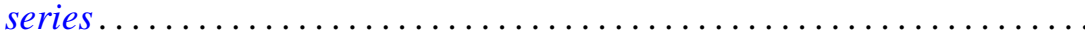

James Guyker, On partial isometries with no isometric part ............

Shigeru Hasegawa and Ryōtarō Satō, A general ratio ergodic theorem for

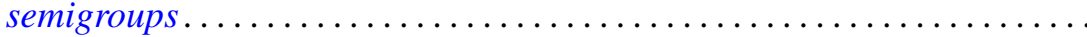

Nigel Kalton and G. V. Wood, Homomorphisms of group algebras with norm less than $\sqrt{2}$.

Thomas Laffey, On the structure of algebraic algebras...

Will Y. K. Lee, On a correctness class of the Bessel type differential operator $S_{\mu}$

Robert D. Little, Complex vector fields and divisible Chern classes ....

Kenneth Louden, Maximal quotient rings of ring extensions . .

Dieter Lutz, Scalar spectral operators, ordered $l^{\rho}$-direct sums, and the

counterexample of Kakutani-McCarthy . .

Ralph Tyrrell Rockafellar and Roger Jean-Baptiste Robert Wets, Stochastic

convex programming: singular multipliers and extended duality

singular multipliers and duality.

Edward Barry Saff and Richard Steven Varga, Geometric overconvergence of rational functions in unbounded domains ..........

Joel Linn Schiff, Isomorphisms between harmonic and P-harmonic Hardy

spaces on Riemann surfaces.

Virinda Mohan Sehgal and S. P. Singh, On a fixed point theorem of

Krasnoselskii for locally convex spaces.

Lewis Shilane, Filtered spaces admitting spectral sequence operations

Michel Smith, Generating large indecomposable continua . 